\title{
Seroprevalence of Specific Antibodies to Treponema pallidum in Blood Donors
}

\author{
Karki S, ${ }^{1}$ Tiwari BR, ${ }^{2}$ Ghimire P, ${ }^{1}$ Maharjan A, ${ }^{2}$ Rajkarnikar $\mathrm{M}^{2}$ \\ ${ }^{1}$ Central Department of Microbiology, Tribhuvan University, ${ }^{2}$ Central Blood Transfusion Service, Nepal Red Cross Society
}

\begin{abstract}
Background: Detection of specific antibodies to Treponema pallidum in serum is correlated with present or past Syphilis. The study aimed to know the seroprevalence of syphilis among blood donors in relation to age, sex, type of donation and times of donation.

Methods: A total of 24,000 blood donors were screened to detect the presence of specific antibodies against T.pallidum by enzyme linked immunosorbent assay (ELISA) according to standard test kit protocol. Initially reactive samples were reconfirmed by repeat testing and repeatedly reactive samples were considered seropositive for Syphilis.

Results: The seroprevalence of specific antibodies to T. pallidum among blood donors was found $0.42 \%(95 \% \mathrm{CI}=$ $0.3-0.5 \%)$. There was an increasing trends of syphilis as the age of the donors increased (Cochrane-armitage test, $\mathrm{P}<$ $0.001)$. The seroprevalence in males was $0.45 \%$ and in females was $0.24 \%(\mathrm{P}>0.05)$, in first time donors was $0.36 \%$ and in repeated donors was $0.48 \%(\mathrm{P}>0.05)$, among volunteer donors was $0.41 \%$ and among replacement donors was $0.50 \%(\mathrm{P}>0.05)$.

Conclusion: The similar seroprevalence in first time and repeat donors as well as in volunteer and replacement donors urges the need of more effective donor education and counselling.
\end{abstract}

Key words: blood donors, seroprevalence, specific treponemal antibodies, syphilis

\section{INTRODUCTION}

In Nepal, prevalence of sexually transmitted infections (STIs) is quite high and estimated to be around $3 \%$ of the population. ${ }^{1}$ The major hospitals of the country report higher prevalence of gonorrhea followed by syphilis, chancroid and herpes genetalis. ${ }^{2}$

Treponema pallidum is a chronic infection with many diverse clinical manifestations that occur in distinct stages. ${ }^{3}$ Syphilis can be transmitted by sexual contact, transplacental transfer and less commonly through indirect routes (contaminated objects, tattoos) and blood transfusions. ${ }^{4,5}$ The treponemal tests have good sensitivity at all stages of disease and indicate either present or past evidence of Syphilis ${ }^{6}$.

The risk of transmission through blood is negligible due to improved donor selection, uniform serologic testing of all blood donors and increased trend of transfusion of refrigerated blood components. ${ }^{7}$ Transmission via blood products is nonetheless theoretically possible since organisms may survive for upto 5 days in blood stored at $4^{\circ} \mathrm{C} .{ }^{3,8}$ In Nepal, the scenario of post transfusion infection is unclear however according to National Center for AIDS and STD Control (NCASC), 24 of total 10,546 officially

Correspondence: Mr. Surendra Karki, Central Department of Microbiology, Tribhuvan University, Kritipur, Nepal. Phone: 9841820025, Email: surendra.karki@ gmail.com 
reported HIV positive cases are described to be associated with blood transfusion or organ transplantation. ${ }^{9}$

The world health organization (WHO) estimates that 12 million new cases of syphilis occur each year and the greatest number of cases was estimated to have occurred in South and Southeast Asia. ${ }^{10}$ In context of Nepal, various studies among high risk individuals have reported that the seroprevalence of Syphilis ranges from 2.5-24.7\%. ${ }^{11,12}$ The seropositivity of syphilis among blood donors in Bhairahava, western Nepal, has been reported to be $0.39 \%$ on the basis of positive RPR test ${ }^{13}$. So far we know, the seroprevalence study of Syphilis using specific treponemal tests among a representative sample of Nepalese blood donors has not been published. This study is expected to be useful in making plans and policies for management of safe blood supply in Nepal and would generate the data regarding seroepidemiology of Syphilis in healthy looking general population.

\section{METHODS}

This was a descriptive cross sectional study conducted in Central Blood Transfusion Service (CBTS), Nepal Red Cross Society (NRCS), Exhibition Road, Kathmandu, from December 1, 2006 to August 1, 2006. Blood donors, donating blood in CBTS or in Mobile camps organized in Kathmandu valley were selected as study population. All blood donors selected for donation as fulfilling per criteria of CBTS were included in the study except those who already donated previously within the specified time period of this study. A total of 24,000 blood donors were included in the present study. Blood donors were tested for detection of specific antibodies against Treponemal pallidum by enzyme linked immunosorbent assay (ELISA) (Enzygnost Syphilis, Dade Behring, Marbug, Germany) in an automated ELISA processor (Behring ELISA processor III, Marbug, Germany). An initial reactive test result was reconfirmed by repeat testing and repeatedly reactive samples were considered seropositive. The data was entered in Microsoft excel spreadsheet collecting the information from Blood donor's form and was analyzed by the statistical software "SPSS ver 11.5". Chi -square test was used for test of association.

Before collecting the blood sample each donor was requested to fill the blood donors form and he/she was assured that the confidentiality would be strictly maintained during testing and recording for mandatory screening of blood for Transfusion transmitted infections as per guidelines of NRCS, CBTS.

\section{RESULTS}

The overall seroprevalence of specific antibodies against Treponema pallidum in Nepalese blood donors was 0.42
\% (95\% Cl=0.3-0.5\%). There was significantly increasing trends in overall seroprevalence as the age increased and the result was consistent for both male and female donors $(P<0.001)$ (Table 1, Figure 1). The mean age of the seropositive donors was 36.56 year (Std.dev=9.283). The seroprevalence was $0.45 \%$ among male donors and $0.24 \%$ among female donors but the difference was not statistically significant $(P>0.05)$. The seroprevalence was higher among repeat donors than among first time donors, but the difference was not statistically significant $(P>0.05)$. Among the 59 seropositive repeat donors, 34 (57.6\%) donated 5 or more times. The seroprevalence was slightly higher among replacement donors than among volunteer donors and the difference was not statistically significant $(P>0.05)$.

\section{DISCUSSION}

This study was conducted to reveal the seroepidemiology of specific antibodies to T. pallidum among Nepalese blood donors. Though the blood donation programs were organized in Kathmandu Valley, donors were from all over the country representing different caste and ethnicity.

Present study revealed that the seroprevalence of specific antibodies to T. pallidum among Nepalese blood donors was $0.42 \%$. A similar seroprevalence was reported from Bhairahava, western Nepal, among blood donors, though nontreponemal test was used for screening in that study. ${ }^{13}$ The seroprevalence observed in present study was much less than in the study reported by Gupta et al. (2004) from India, Rahman et al. (2002) from Lahore, Pakistan, Matee et al. (2006) from Tanzania, and Butsashvili et al. (2001) from Georgia. ${ }_{14{ }^{17}}$. The seroprevalence observed in present study was slightly higher than the seroprevalence reported by Moiz et al. (2004) from Pakistan and Gunduz et al. ${ }^{18}$ (2005) from Turkey, the lower seroprevalence rate in these study was possibly due to the screening by non treponemal tests, as nontreponemal tests lack sensitivity at later stage of the disease and become negative in successfully treated patients. ${ }^{19,20}$ Using the specific treponemal screening test Garcia Montalvo has reported a slightly lower seropositivity among Mexican blood donors than in our present study. ${ }^{21}$

The study revealed considerably higher seroprevalence of syphilis among the male donors, though the difference was not statistically significant. The seroprevalence rate observed for male donors in present study was lower than in the study reported by Joshi et al. in a similar population but in that study RPR was used for screening. ${ }^{22}$

The study showed that there was increasing trends in seroprevalence of syphilis as the age of the donors increased and the result was consistent with both 


\begin{tabular}{|c|c|c|c|c|}
\hline Blood donors & Total (no) & Number of Seropositive & Seroprevalence (\%) & $P$ - value \\
\hline Total donors & 24,000 & 102 & 0.43 & \\
\hline \multicolumn{5}{|l|}{ Sex } \\
\hline Male & 21,089 & 95 & 0.45 & \multirow{2}{*}{$>0.05$} \\
\hline Female & 2,911 & 7 & 0.24 & \\
\hline \multicolumn{5}{|l|}{ Age } \\
\hline$\leq 20$ & 3,838 & 5 & 0.13 & \multirow{5}{*}{$\begin{array}{l}P \text { for trends } \\
\text { (Cochrane-armitage test) } \\
<0.0001\end{array}$} \\
\hline $21-30$ & 11,500 & 23 & 0.20 & \\
\hline $31-40$ & 6,051 & 40 & 0.66 & \\
\hline $41-50$ & 2,235 & 26 & 1.16 & \\
\hline $51-60$ & 376 & 8 & 2.12 & \\
\hline \multicolumn{5}{|c|}{ Times of donation: } \\
\hline First time & 11,907 & 43 & 0.36 & \multirow[b]{2}{*}{$>0.05$} \\
\hline Repeated & 12,093 & 59 & 0.48 & \\
\hline \multicolumn{5}{|c|}{ Type of donation: } \\
\hline \multirow{2}{*}{$\begin{array}{r}\text { Volunteer } \\
\text { Replacement }\end{array}$} & 21,443 & 89 & 0.41 & \multirow{2}{*}{$>0.05$} \\
\hline & 2,557 & 13 & 0.50 & \\
\hline
\end{tabular}

$\mathrm{P}$ for trends in male donors $<0.001$ and $\mathrm{P}$ for trends in female donors $<0.001$ (Cochrane-armitage test for trends).

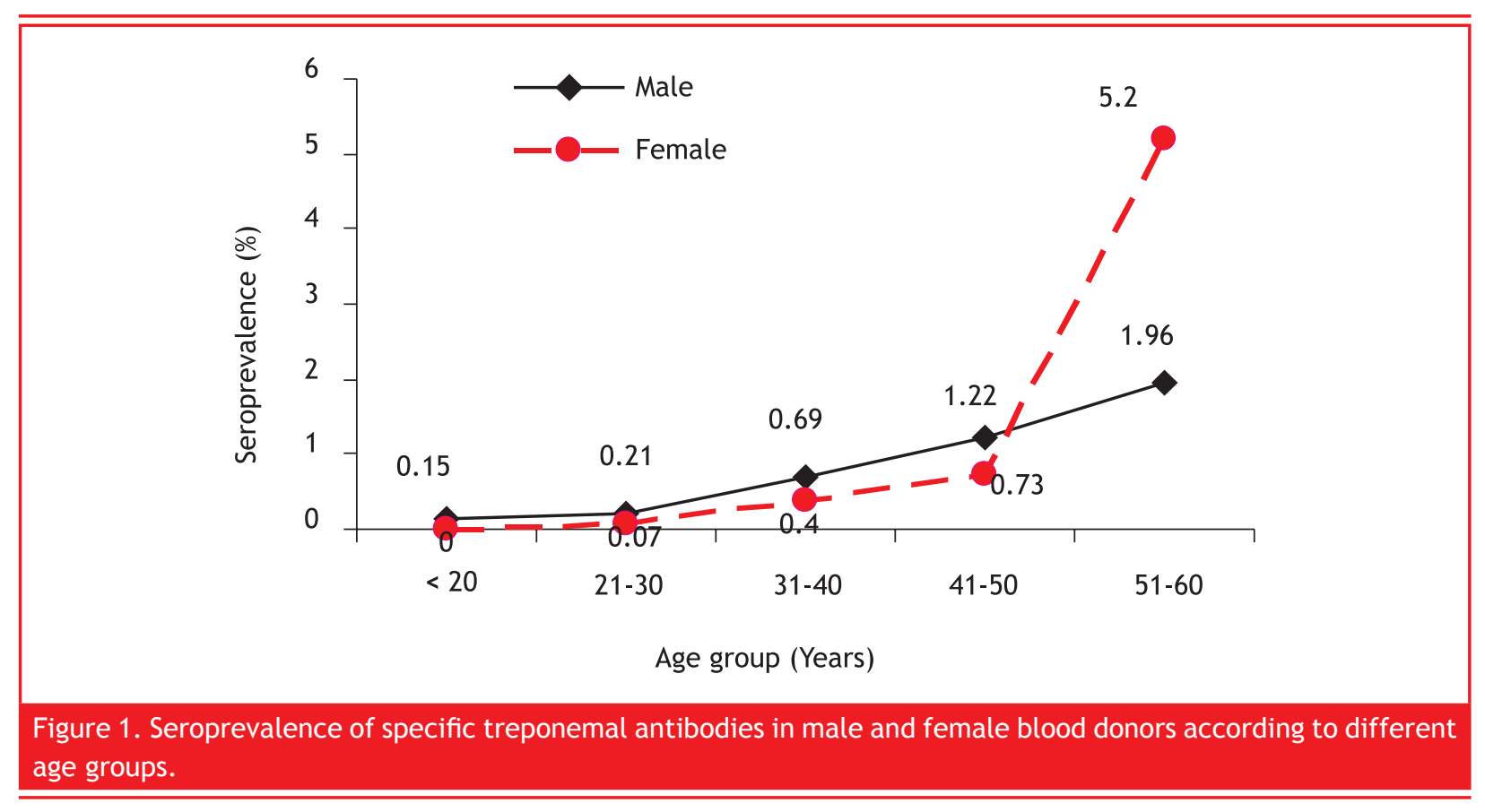

male and female donors, a characteristic of sexually transmitted infections.

Regular repeated donors are considered the safer blood donor groups and such type of donation has been encouraged worldwide. Though statistically non significant, considerably higher seroprevalence rate among repeat blood donors revealed by present study was notable. This was possibly due to the lack of CBTS initiated donor notification and counseling of the TTIs seropositive donors as well as might be due to the ineffectiveness of donor education and counseling. 
Present study has revealed statistically non significant difference in the seroprevalence of syphilis among replacement and volunteer donors though the seroprevalence was slightly higher in replacement donations, which was similar to the study published by Matte et al. from Tanzania and Mbanya et al. from Cameroon ${ }^{16,23}$. Interestingly, present study has revealed totally contrasting result compairing to the results reported by Chander et al, which reported significantly higher seroprevalence among voluntary donors. ${ }^{13}$ Such difference might be due to a large difference in sample size.

\section{CONCLUSION}

The seroprevalence of specific antibodies to T. pallidum, $0.42 \%$ observed among blood donors was relatively lower as compared to other studies in Asian and African countries. However, the similar seroprevalence in first time and repeat donors as well as in volunteer and replacement donors urges the need of more efficient donor education and counselling. In order to improve the blood safety, donor notification and counseling for TTIs positive donor might prove valueable. So, the suitability and feasibility study for donors' notification and counselling of TTIs positive donors is recommended.

\section{ACKNOWLEDGMENTS}

The authors would like to acknowledge Nepal Red Cross Society for its support to conduct this study.

\section{REFERENCES}

1. Subedi BK. Sexually transmitted diseases in Nepal. JNMA 1996;34:277-8.

2. Dixit H. Nepal's Quest for Health. 3rd ed. Kathmandu: Educational publishing house; 2005 . p. 28-9

3. Lafond RE, Lukehart SA. Biological basis for syphilis. Clinical Microbiology Reviews 2006;19:29-9.

4. Avelleira JCR, Bottino G. Syphilis: diagnosis, treatment and control. An Bras Dermatol 2006;81:111-6..

5. Van der Sluis JJ, Ten kate FJ, Vujevski VD, Kothe FC, Albers GM, Van Eijk RV. Transfusion syphilis, survival of Treponema pallidum in donor blood II. Dose dependence of experimentally determined survival times. Vox Sang 1985;49:390-9.

6. Winn W, Allen S, Janda W, Koneman E, Procop G, Schreckenberger $\mathrm{P}$ et al. Koneman's Color Atlas and Textbook of Diagnostic Microbiology. 6th ed. Lippincots Williams and Wilkins; 2006.

7. Garnett GP, Aral SO, Hoyle DV, Cates W Jr, Anderson RM. The natural history of Syphilis. Implications for the transfusion dynamics and control of infection. Sex Transm Dis 1997;24:18590 .
8. Willcox RR, Guthe T. Treponema pallidum, A bibliographical review of the morphology, culture and survival of T. pallidum and associated organism. Bull WHO 1966;35: 1-169.

9. National Center for AIDS and STD Control. Cumulative HIV and AIDS situation of Nepal. Final report. Kathmandu: National Center for AIDS and STD Control; 2007.

10. Gerbase AC, Rowley JT, Heymann DH, Berkley SF, Piot P. Global prevalence and incidence estimates of selected curable STDs. Sex. Transm. Infect 1998;74:S12-S16.

11. Family Health International. Kathmandu FSW Seroprevalence Study (2001). [Online]. 2001 [cited 2008 Jan 10]. Available from: URL:http://www.fhi.org/en/HIVAIDS/pub/survreports/ kathmandu.htm

12. Poudel KC, Okumura J, Serchand JB, Jimba M, Murakami I. Mumbai disease in far western Nepal: HIV infection and Syphilis among male migrant returnees and non migrants. Tropical Medicine and Int Health 2003;8:933-9.

13. Chander A, Pahwa VK. Status of infectious disease markers among blood donors in a teaching hospital, Bhairahawa, western Nepal. J Commun Dis 2003;35:188-7.

14. Gupta N, KumarV, Kaur A. Seroprevalence of HIV, HBV, HCV and syphilis in voluntary blood donors. Indian J Med Sci 2004;58:2557.

15. Rahman M, Akhtar GM, LodhiY. Seroprevalence of Syphilis in the blood donors in Lahore. Pak J Med Sci 2002;18:284-6.

16. Matee MI, Magesa PM, Lyamuya EF. Seroprevalence of human immunodeficiency virus, hepatitis B and $\mathrm{C}$ viruses and syphilis infections among blood donors at the Muhimbili National Hospital in Dar Es Salaam, Tanzania. BMC Public Health 2006;6:21.

17. Butsashvili M, Tsertsvadze T, McNutt LA, Kamkamidze G, Gvetadze R Badridze N. Prevalence of hepatitis B, hepatitis C, syphilis and HIV in Georgian blood donors. European Journal of Epidemiology 2001;17:693-5.

18. Moiz B, Adil SN, Khursid M. Seroprevalence of Syphilis in Healthy noncommercial blood donors in Karachi. JCPSP 2006;16:385-6.

19. GunduzT, Mumcuoglu I, Guray M. Evaluation of hepatitis B surface antigen, anti-hepatitis $\mathrm{C}$ virus and anti-human immunodeficiency virus antibodies and syphilis seropositivity in blood donors: six years' seropositivity. Int J Clin Pharma Res 2005;25: 155-8.

20. Larsen SA, Steiner BM, Rudolph AH. Laboratory diagnosis and Interpretation of Tests for Syphilis. Clin Microbiol Review $1995 ; 8: 1-21$

21. Garcia Montalvo BM. Seropositivity of HIV, HBV, HCV and Treponema pallidum in blood donors in southeast Mexico. Rev Invest Clin 2006;58:567-2.

22. Joshi SK, Ghimire GR. Serological prevalence of antibodies to human immunodeficiency virus (HIV) and hepatitis B virus (HBV) among healthy Nepalese males--a retrospective study. KUMJ $2003 ; 1: 251-5$

23. Mbanya DN ,Takam D, Ndumbe PM. Serological findings among first time blood donors in Yaounde, Cameroon: Is safe donation a reality or a myth? Transfusion Medicine 2003; 13:267-3. 